\title{
Transparent Boundary Conditions for Wave Propagation on Unbounded Domains
}

\author{
Dorin-Cezar Ionescu ${ }^{1}$ and Heiner Igel ${ }^{2}$ \\ 1 Queensland University Advanced Centre for Earthquake Studies (QUAKES) \\ The University of Queensland, Brisbane, QLD 4072, Australia \\ 2 Department für Geo- und Umweltwissenschaften, Ludwig-Maximilians-Universität \\ Theresienstr. 41, 80333 München, Germany
}

\begin{abstract}
The numerical solution of the time dependent wave equation in an unbounded domain generally leads to a truncation of this domain, which requires the introduction of an artificial boundary with associated boundary conditions. Such nonreflecting conditions ensure the equivalence between the solution of the original problem in the unbounded region and the solution inside the artificial boundary. We consider the acoustic wave equation and derive exact transparent boundary conditions that are local in time and can be directly used in explicit methods. These conditions annihilate wave harmonics up to a given order on a spherical artificial boundary, and we show how to combine the derived boundary condition with a finite difference method. The analysis is complemented by a numerical example in two spatial dimensions that illustrates the usefulness and accuracy of transparent boundary conditions.
\end{abstract}

\section{Introduction}

Modern trends in the development of numerical methods lead to higher and higher requirements for computational accuracy. The numerical solution of the wave equation on unbounded domains requires a truncation to fit the infinite region on a finite computer. Minimizing the amount of spurious reflections requires in many cases the introduction of an artificial boundary and of associated boundary conditions. The critical importance of these techniques becomes particularly evident when one considers that the gains made in the computational domain by using sophisticated high-order numerical approaches may vanish to a large extent as result of violating the conditions at the artificial boundary.

Despite the computational speed of finite difference schemes and the robustness of finite elements in handling complex geometries the resulting numerical error consists of two independent contributions: the discretization error of the numerical method used and the spurious reflection generated at the artificial boundary. This spurious contribution travels back and substantially degrades the accuracy of the solution everywhere in the computational domain. Unless both error components are reduced systematically, the numerical solution does not converge to the solution of the original problem in the infinite region.

There are various techniques for the approximate handling of boundary conditions at the external boundary of a finite domain constructed from the original 
unbounded domain by means of truncation. One class of conditions is given by local differential operators [2], [3], including conditions that perfectly annihilate impinging waves at a finite number of selected angles of incidence [4], whilst a different approach is based on absorbing layers [5]. There are cases, where some of the difficulties with boundary conditions may be avoided partially by using a momentum space approach [6]. In contrast to grid methods in coordinate space where continuum waves spread over the entire space, in momentum space the waves are confined to a small finite volume and the dynamics stays localized around the origin at all times.

Exact nonreflecting boundary conditions that are nonlocal in both space and time have been investigated numerically in [7]. Numerical methods based on these exact conditions display a long-time instability, and a major disadvantage is related to the nonlocal character of the boundary condition in time. Due to the temporal nonlocality that requires information from previous time steps, these methods require a considerably longer computational time than explicit schemes for the wave equation. Recently, very important progress has been made by Grote and Keller [8] by deriving exact nonreflecting boundary conditions that are local in time.

In the present work we study exact transparent boundary conditions that are local in time for the scalar wave equation for the general case in two and three spatial dimensions. In contrast to [8] where an integral equation is used, our approach is based on the separation of variables combined with recurrence relations that provide a very direct derivation of the boundary condition. Since the derived condition is local in time and is equivalent to the result obtained in [8], our formulation complements the integral transform approach and is expected to have applications to cases where the latter method is difficult to apply.

The local character in time of the boundary condition and its explicit representation that requires only first order derivatives of the solution makes it relatively easy to apply in calculations based on explicit schemes. In contrast to earlier studies based on exact but nonlocal boundary conditions, the present finite differences implementation does not require a significant increase in the computational time. This highlights a key point in practical applications of such conditions in that the related implementations should not become computationally too expensive. In Sec. 2, we illustrate the fundamental ideas underlying the derivation of nonreflecting boundary conditions for the one-dimensional case and present the extension to higher dimensions. In Sec. 3 we discuss the implementation in a finite differences scheme and present a simple two dimensional numerical example that illustrates the usefulness of transparent boundary conditions. The conclusions of the present study are given in Sec. 4 .

\section{Theoretical Approach}

\subsection{The One-Dimensional Wave Equation}

We consider the one-dimensional wave equation describing the propagation of perturbations along the positive real axis $(x \geq 0, t \geq 0)$ with velocity $c=1$ that 
are induced by a general and possibly nonlinear forcing term $f=f\left(x, t, \Phi, \partial_{x} \Phi\right)$

$$
\left(\partial_{t}^{2}-\partial_{x}^{2}\right) \Phi(x, t)=f,
$$

where $\Phi(x, t)$ represents the displacement of an infinitely long string and $\partial_{t}=$ $\partial / \partial t$. Upon requiring $\Phi(0, t)=0$ for the state at rest we assume $\Phi(x, t)$ to describe the position of a string fixed at the origin. We define the initial conditions by the string position and velocity at $t=0$ by $\Phi(x, 0)=U_{0}$ and $\left.\partial_{t} \Phi(x, t)\right|_{t=0}=V_{0}$.

The local character of the problem is defined by assuming $f=0$ for $x \geq$ $L, \forall t \geq 0$. Thus, the positive $x$-axis separates into two distinctly different regions: the bounded (interior) domain $x \leq L$, and the unbounded (exterior) region $x \geq L$ where the forcing term $f$ vanishes. The two regions are separated by the artificial boundary at $x=L$.

To find the exact absorbing boundary condition at $x=L$ it is useful to separate outgoing from incoming waves by defining

$$
v=\partial_{t} \Phi+\partial_{x} \Phi, \text { and } \quad w=\partial_{t} \Phi-\partial_{x} \Phi .
$$

Since $\Phi(x, t)$ is a solution of Eq. (1) for $x \geq L$, i.e. $\left(\partial_{t}^{2}-\partial_{x}^{2}\right) \Phi=0$, in the exterior region from (2)

$$
\begin{aligned}
0 & =\left(\partial_{t}-\partial_{x}\right)\left[\left(\partial_{t}+\partial_{x}\right) \Phi\right]=\left(\partial_{t}-\partial_{x}\right) v, \\
0 & =\left(\partial_{t}+\partial_{x}\right)\left[\left(\partial_{t}-\partial_{x}\right) \Phi\right]=\left(\partial_{t}+\partial_{x}\right) w .
\end{aligned}
$$

This system of first-order equations has the general solution

$$
v(x, t)=\psi(x+t), \quad \text { and } \quad w(x, t)=\varphi(x-t),
$$

where $\psi$ and $\varphi$ are arbitrary functions that are determined by initial and boundary conditions. Thus, incoming $(v)$ and outgoing $(w)$ waves are defined as

$$
\begin{aligned}
& v(x, t)=\text { const., for } x+t=\text { const. (incoming) } \\
& w(x, t)=\text { const., for } x-t=\text { const. (outgoing). }
\end{aligned}
$$

Since there are no incoming waves in the exterior region, it follows $v(L, t)=0$. The requirement of a purely outgoing wave for $x \geq L$ combined with the definition for incoming waves $v$ from eq. (2) yields the exact nonreflecting boundary condition for the displacement $\Phi(x, t)$

$$
\left.\left(\partial_{t}+\partial_{x}\right) \Phi(x, t)\right|_{x=L}=0 .
$$

This expression which is local in time guarantees that the artificial boundary at $x=L$ is perfectly transparent to both incoming and outgoing waves as they leave the interior region without any spurious reflection. Note that since the derivation of the exact boundary condition (6) depends solely on properties in the exterior domain, $x \geq L$, the problem inside the computational volume can be arbitrarily complex. 


\subsection{Transparent Boundary Conditions in Higher Dimensions}

The derivation of exact absorbing boundary conditions in higher dimensions is considerably more challenging as compared to the one dimensional case discussed previously. Distinctly different from the one-dimensional case where waves can propagate in two directions only, in two and more dimensions waves propagate in infinitely many directions.

In the following we consider wave propagation in an unbounded region $\mathbb{R}^{3}$ and surround the computational region $\tilde{\Omega}$ containing the forcing term $f$ by an artificial boundary $S$ that is assumed to be the surface of a sphere with radius $R$. In the exterior domain one has $f=0$, and the wave function $\psi(\mathbf{r}, t)$ satisfies the homogeneous wave equation with propagation velocity $c>0$, i.e.

$$
\left(\frac{1}{c^{2}} \partial_{t}^{2}-\Delta\right) \psi(\mathbf{r}, t)=0 \text { in } \mathbb{R}^{3} \backslash \tilde{\Omega},
$$

with initial conditions $\psi(\mathbf{r}, 0)=0$ and $\partial_{t} \psi(\mathbf{r}, 0)=0$. It is useful to separate the variables by expanding the solution in spherical coordinates $r, \vartheta, \varphi$

$$
\psi(\mathbf{r}, t)=\sum_{l=0}^{\infty} \sum_{m=-l}^{l} \psi_{l, m}(r, t) Y_{l, m}(\vartheta, \varphi),
$$

where the spherical harmonics

$$
Y_{l, m}(\vartheta, \varphi)=\sqrt{\frac{(2 l+1)(l-|m|) !}{4 \pi(l+|m|) !}} P_{l}^{|m|}(\cos \vartheta) e^{i m \varphi},
$$

are orthonormal and the functions $P_{l}^{|m|}$ are Legendre polynomials. Using the orthonormality properties of the $Y_{l, m}$ the radial time-dependent functions $\psi_{l, m}(r, t)$ may be written as

$$
\psi_{l, m}(r, t)=\int_{0}^{\pi} d \vartheta \sin \vartheta \int_{0}^{2 \pi} d \varphi Y_{l, m}^{*}(\vartheta, \varphi) \psi(r, \vartheta, \varphi, t) .
$$

By inserting expression (8) in eq. (7) one obtains the radial equation

$$
\left[\frac{1}{c^{2}} \partial_{t}^{2}-\partial_{r}^{2}-\frac{2}{r} \partial_{r}+\frac{l(l+1)}{r^{2}}\right] \psi_{l, m}(r, t)=0,
$$

with the initial conditions $\psi_{l, m}(r, 0)=0$ and $\partial_{t} \psi_{l, m}(r, 0)=0$. The differential operator in the square brackets which we denote by $R_{l}$, satisfies a remarkable commutation-like relation $R_{l}\left[\partial_{r}-(l-1) / r\right]=\left[\partial_{r}-(l-1) / r\right] R_{l-1}$, or

$$
R_{l}\left(\partial_{r}-\frac{l-1}{r}\right) \phi_{l-1}(r, t)=\left(\partial_{r}-\frac{l-1}{r}\right) R_{l-1} \phi_{l-1}(r, t)=0,
$$

whenever $R_{l-1} \phi_{l-1}=0$. Thus, one obtains a solution of the $l$-th order equation $R_{l} \phi_{l}=0$ from a $(l-1)$ th order solution according to the recurrence relation

$$
\phi_{l}(r, t)=\left[\partial_{r}-\frac{l-1}{r}\right] \phi_{l-1}(r, t),
$$


that can be also obtained by use of properties of spherical Bessel functions [10]. Recursive use of (13) yields a representation for the radial functions $\psi_{l, m}$

$$
\psi_{l, m}=\left[\partial_{r}-\frac{l-1}{r}\right]\left[\partial_{r}-\frac{l-2}{r}\right] \phi_{l-2, m}=\ldots=\prod_{i=1}^{l}\left[\partial_{r}-\frac{i-1}{r}\right] \phi_{l, m}
$$

where $\phi_{l, m}$ is a solution to the $l=0$ version of eq. (11). It follows that the modified radial function $\Phi_{l, m}(r, t)=r \phi_{l, m}$ satisfies a simple one-dimensional wave equation, i.e.

$$
\left(\frac{1}{c^{2}} \partial_{t}^{2}-\partial_{r}^{2}\right) \Phi_{l, m}(r, t)=0
$$

As shown in Sec. 2.1, a general solution for outgoing waves is written as $\Phi(r-c t)$, such that for $l \geq 1$ the radial functions $\psi_{l, m}$ are expressed as

$$
\psi_{l, m}(r, t)=\prod_{i=1}^{l}\left(\partial_{r}-\frac{i-1}{r}\right) \frac{1}{r} \Phi_{l, m}(r-c t),
$$

where the index denotes a radial wave function of order $l$. Recursive use of this relations enables one after some rearrangement to rewrite the $l$-th order radial function as a sum over $l$, i.e.

$$
\begin{aligned}
\psi_{l, m}(r, t) & =\sum_{i=0}^{l} \frac{(-)^{i}}{r^{i+1}} \rho_{l, i} \frac{\partial^{l-i}}{\partial r^{l-i}} \Phi_{l, m}(r-c t) \\
& =(-)^{l} \sum_{i=0}^{l} \frac{1}{c^{l-i} r^{i+1}} \rho_{l, i} \frac{\partial^{l-i}}{\partial t^{l-i}} \Phi_{l, m}(r-c t),
\end{aligned}
$$

where $\rho_{l, i}=(l+i) ! /\left[2^{i} i !(l-i) !\right]$. These coefficients can be obtained by using induction in eq. (17) to obtain recurrence relations for $\rho_{l, i}$, and we replaced the spatial derivative with a time derivative using

$$
(-)^{k} \frac{\partial^{k}}{\partial r^{k}} \Phi_{l, m}(r-c t)=\frac{1}{c^{k}} \frac{\partial^{k}}{\partial t^{k}} \Phi_{l, m}(r-c t) .
$$

In analogy with ref. [8] we now replace the radial derivative with a time derivative by applying the operator $B_{1}$ on the radial function

$$
B_{1} \psi_{l, m}=\left[\partial_{r}+\frac{1}{c} \partial_{t}+\frac{1}{r}\right] \psi_{l, m}=\frac{(-)^{l+1}}{r} \sum_{i=0}^{l} \frac{i \rho_{l, i}}{c^{l-i} r^{i+1}} \frac{\partial^{l-i}}{\partial t^{l-i}} \Phi_{l, m}(r-c t),(1
$$

where we used eq. (17). Finally, using expansion (8) and multiplying the last expression by $Y_{l m}$ with summation over $l$ and $m$, we obtain

$$
B_{1} \psi(R, \vartheta, \varphi, t)=-\frac{1}{R} \sum_{l, m} Y_{l, m}(\vartheta, \varphi) \sum_{i=1}^{l} \frac{(-)^{l} i \rho_{l i}}{c^{l-i} R^{i+1}} \frac{\partial^{l-i}}{\partial t^{l-i}} \Phi_{l, m}(R-c t)
$$


In the general case, the functions $\Phi_{l, m}$ are obtained by evaluating Eq. (17) at $r=R$. Since $\rho_{l 0}=1$ this leads to the solution of a linear differential equation

$$
\frac{1}{c^{l}} \frac{d^{l}}{d t^{l}} \tilde{\Phi}_{l, m}(t)=-\sum_{i=1}^{l} \frac{\rho_{l i}}{c^{l-i} R^{i}} \frac{d^{l-i}}{d t^{l-i}} \tilde{\Phi}_{l, m}(R-c t)+\psi_{l, m}(R, t),
$$

where we have substituted $\tilde{\Phi}_{l, m}=(-)^{l} \Phi_{l, m} / R$ and the inhomogeneous term $\psi_{l, m}(R, t)$ is given by Eq. (10) evaluated at $r=R$.

Expression (20) represents the exact nonreflecting boundary condition in the form obtained in [8] where integral transforms formed the basis of the approach. Note that in practical calculations truncation of the summation over $l$ at a finite value $l=L$ leads to an exact representation of modes with $l \leq L$. Thus, the boundary condition reduces to $\left.B_{1} \psi\right|_{r=R}=0$ for harmonic modes with $l^{\prime}>$ $L$. In particular, $\left.B_{1} \psi\right|_{r=R}=0$ is an exact boundary condition for spherically symmetric modes $(l=0)$.

\section{$3 \quad$ Numerical Results Using Finite Differences}

Using the results of the previous section, we illustrate the use of transparent boundary conditions and their numerical implementation using a finite difference formulation. The wave equation is discretized both in space and time using centred finite differences. At time $t_{k}=k \Delta t$, we denote by $\Psi^{k}(n)$ the numerical approximation to the time dependent wave function $\psi(\mathbf{r}, t)$ and by $f^{k}(n)$ the forcing term at the $n$-th grid point $r_{n}$ in radial direction [8]. The numerical solution is advanced in time using

$$
\Psi^{k+1}(n)=2 \Psi^{k}(n)-\Psi^{k-1}(n)+(\Delta t)^{2}\left[\mathcal{D} \Psi^{k}(n)+f^{k}(n)\right],
$$

where $\mathcal{D}$ represents a finite difference approximation to the Laplace operator $\Delta$.

An apparent complication occurs when the Laplace operator is to be calculated at the outer most radial grid point, $r_{n}=R$, since this calculation uses values of $\Psi^{k}(n+1)$ belonging to the exterior region. However this problem can be solved by using the explicit representation of the derived boundary condition. More precisely, one obtains an additional relation between the quantities $\Psi^{k+1}(n)$ and $\Psi^{k}(n+1)$ by using a finite difference representation of the boundary condition equation (20) at $r_{n}=R$. Consequently, the problem is solved by coupling the two equations for $\Psi^{k+1}(n)$ and $\Psi^{k}(n+1)$, allowing one to solve for $\Psi^{k+1}(n)$. Due to the local character in time of the boundary condition, both the differential equation and the boundary condition are discretized in time about $t=t_{k}$, and only function values at $t=t_{k}$ are needed in a given time step [8].

This procedure becomes particularly simple and is best illustrated using a cartesian grid $x_{n}=n \Delta x$ in one dimension. The second space derivative $\partial_{x}^{2}$ is written as

$$
\mathcal{D} \Psi^{k}(n)=\frac{\Psi^{k}(n+1)-2 \Psi^{k}(n)+\Psi^{k}(n-1)}{(\Delta x)^{2}}
$$


and using the finite difference representation of the boundary condition we arrive after some algebra at

$$
\Psi^{k}(n+1)=-\frac{1}{c} \frac{\Delta x}{\Delta t}\left[\Psi^{k+1}(n)-\Psi^{k-1}(n)\right]+\Psi^{k}(n-1)
$$

which represents the additional equation for $\Psi^{k+1}(n)$. By combining the two equations for $\Psi^{k+1}(n)$ and $\Psi^{k}(n+1)$ one finds

$$
\Psi^{k+1}(n)=\frac{2 \Psi^{k}(n)+(\alpha-1) \Psi^{k-1}(n)+2 \alpha^{2}\left[\Psi^{k}(n-1)-\Psi^{k}(n)\right]}{1+\alpha}
$$

where $\alpha=c \Delta t / \Delta x$. This expression clearly shows that the evaluation of the time extrapolated function $\Psi^{k+1}(n)$ at the boundary does not depend on function values located outside the computational domain.

Using the approach described above we are now in a position to analyse the time evolution of perturbations using transparent boundary conditions. In Figures 1 and 2 we display snapshots of the two-dimensional wave function at different times for $t=82,128,270,450$, and 900, respectively. The contour plots on the right hand side of the figures display the position of time evolved waves in the $x y$-plane, where the computational domain extends from an inner radius $r_{<}=1000 \mathrm{~km}$ to the outer radius $r_{>}=6371 \mathrm{~km}$, and we used $\Delta t=2.5 \mathrm{~s}$, $d r \simeq 3.5 \mathrm{~km}$, and $c=5 \mathrm{~km} / \mathrm{s}$. The plots on the left show the dependence of the associated wave function on the radial coordinate $r$. While the starting point of the calculation is $t=0$, the perturbing source is assumed to be proportional to a Gaussian $\exp \left(t-t_{0}\right)^{2}$ and is located on a circle with $r_{0} \simeq 4700 \mathrm{~km}$. This may be associated with an explosive point source that is located at the origin $r_{<}=r_{0} \rightarrow 0$ in the far past. For $t=82$ we observe a strong peak located in the vicinity of the source. In the contour plot on the right hand side, one sees that the wave is located in the area between the two bright circles.

For larger times, at $t=128$, the wave separates in two independent contributions propagating in opposite directions along the radial coordinate $r$. While one of the waves moves outwards towards increasing $r$-values the other wave propagates inwards, as indicated by the two arrows in the left figure. Note that the amplitude decreases in magnitude with increasing $r$-values as a result of larger surface elements $r d r d \varphi$. At $\mathrm{t}=270$, it is seen that only the ingoing wave can be found inside the computational region as the outgoing one passes the artificial boundary ar $r=R$ without any reflection. On the other hand, the ingoing wave propagating towards smaller $r$-values changes its sign and the direction of propagation after encountering the margin $r_{<}$of the inner circle. As a result, for larger times $(t=450)$ this wave propagates towards the margin of the computational domain but with opposite sign. Finally, for even larger times this wave passes the artificial boundary at $r=R$ without any reflection. Thus, for $t=900$ the computational region is seen to be completely unperturbed and, as a result of the boundary condition, the artificial boundary appears perfectly transparent to the wave as there is no spurious reflection. 

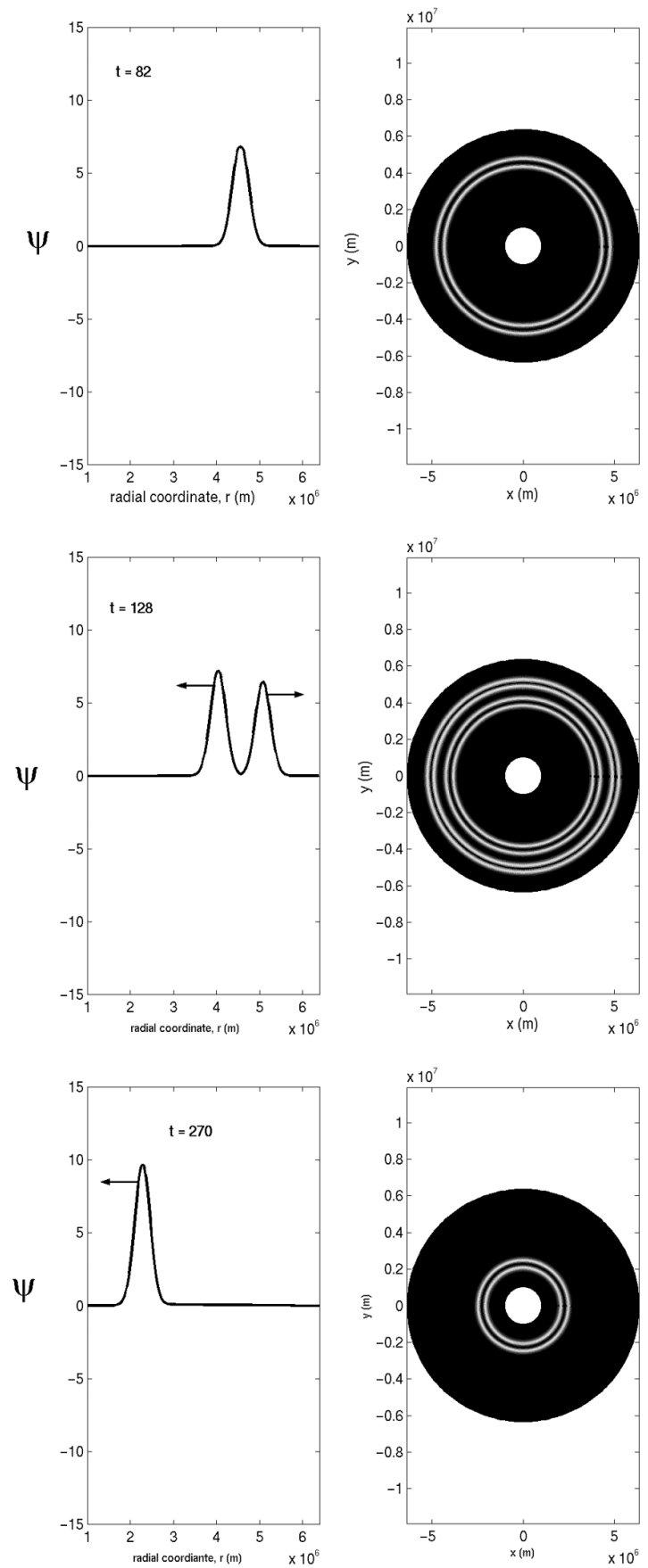

Fig. 1. Snapshots of the time evolved wave function obtained by the numerical solution of the wave equation incorporating the nonreflecting boundary condition for $t=82,128$, and $t=270$. The initial wave separates in two parts propagating in opposite directions. 

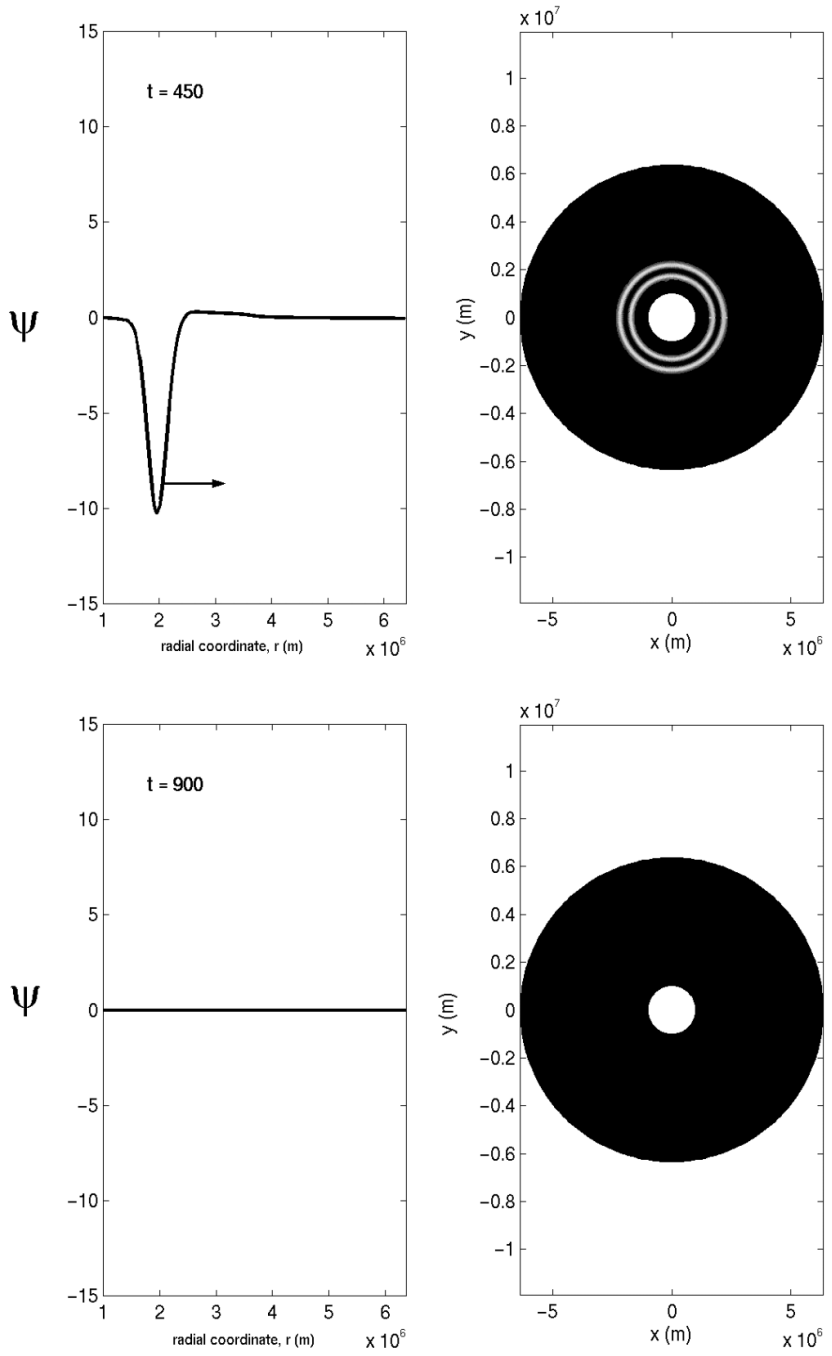

Fig. 2. The same as in Fig. 1 for larger times $t=450$, and 900. At large asymptotic times both components leave the computational domain and no reflection at the artificial boundary is observed. 


\section{Concluding Remarks}

We investigate exact nonreflecting boundary conditions that are local in time for the acoustic wave equation in two and three dimensions. The present approach is based on a separation of variables combined with recurrence relations that provide a very direct derivation of the boundary condition. This formulation can be expected to have applications to situations where other methods are difficult to apply or even impracticable. By adopting an alternative point of view for attacking such problems, the present methodology complements the integral transform approach, thus extending and enhancing the strength of the theory for deriving exact nonreflecting boundary conditions.

The derived boundary condition requires only first order derivatives of the solution which makes it relatively easy to use in explicit schemes. Using finite differences the time extrapolation of the solution and the calculation of the spatial derivatives require unknown function values that lie outside the computational domain. This apparent complication is solved, and by using a simple numerical example in two dimensions we show how these exterior values can be eliminated.

Finally, we emphasize that the derivation of the boundary condition depends only on the behaviour in the exterior domain, such that the problem inside the computational region can be arbitrarily complex, e.g. nonlinear. Furthermore, as there is no unphysical reflection at the artificial boundary associated with the computational region, the derived condition ensures perfect transparency that leads to a long-time stability of the numerical scheme. After this work was completed we learned about a similar approach used in [9].

\section{Acknowledgement.}

This work was supported in part by the University of Queensland under Grant No. UQRSF 2002001336, by the Australian Research Council, by the Australian Computational Earth Systems Simulator (ACcESS) Major National Research Facility, and by the International Quality Network (IQN) Georisk Program at the University of München.

\section{References}

1. Tsynkov, S.V.: Appl. Num. Math. 27, 465 (1998).

2. Clayton, R.W. and Engquist, B.: Bull. Sei. Soc. Am. 67, 1529 (1977).

3. Engquist, B. and Majda, A.: Commun. Pure Appl. Math. 32, 313 (1979).

4. Higdon, R.L.: SIAM J. Num. Anal. 27, 831 (1990).

5. Israeli, M. and Orszag, S.A.: J. Comput. Phys. 41, 115 (1981).

6. Ionescu, D.C. and Belkacem, A.: Eur. Phys. Journal D 18, 301 (2002); see also Phys. Scripta T80, 128 (1999).

7. Givoli, D. and Cohen, D.: J. Comput. Phys. 117, 102 (1995).

8. Grote, M.J. and Keller, J.B.: SIAM J. Appl. Math. 55, 280 (1995); and 60, 803 (2000); see also J. Comput. Phys. 139, 327 (1998).

9. Thompson, L. and Huan, R.: submitted to Comp. Meth. Appl. Mech. Eng. (2003).

10. Abramowitz, M. and Stegun, I.A.: Handbook of Mathematical Functions (Harri Deutsch, Thun, Frankfurt am Main, 1984). 